\title{
NRAGE, a p75NTR adaptor protein, is required for developmental apoptosis in vivo
}

\author{
MJM Bertrand ${ }^{1,2,3}$, RS Kenchappa ${ }^{4}$, D Andrieu ${ }^{5}$, M Leclercq-Smekens ${ }^{1}$, HNT Nguyen ${ }^{1}$, BD Carter ${ }^{4}$, F Muscatelli ${ }^{5}$, PA Barker ${ }^{*, 3,6}$ \\ and $O$ De Backer ${ }^{1,2,6}$
}

NRAGE (also known as Maged1, DIxin) is a member of the MAGE gene family that may play a role in the neuronal apoptosis that is regulated by the $\mathrm{p75}$ neurotrophin receptor (p75NTR). To test this hypothesis in vivo, we generated NRAGE knockout mice and found that NRAGE deletion caused a defect in developmental apoptosis of sympathetic neurons of the superior cervical ganglia, similar to that observed in p75NTR knockout mice. Primary sympathetic neurons derived from NRAGE knockout mice were resistant to apoptosis induced by brain-derived neurotrophic factor (BDNF), a pro-apoptotic p75NTR ligand, and NRAGEdeficient sympathetic neurons show attenuated BDNF-dependent JNK activation. Hair follicle catagen is an apoptosis-like process that is dependent on p75NTR signaling; we show that NRAGE and p75NTR show regulated co-expression in the hair follicle and that identical defects in hair follicle catagen are present in NRAGE and p75NTR knockout mice. Interestingly, NRAGE knockout mice have severe defects in motoneuron apoptosis that are not observed in p75NTR knockout animals, raising the possibility that NRAGE may facilitate apoptosis induced by receptors other than p75NTR. Together, these studies demonstrate that NRAGE plays an important role in apoptotic-signaling in vivo.

Cell Death and Differentiation (2008) 15, 1921-1929; doi:10.1038/cdd.2008.127; published online 5 September 2008

The p75 neurotrophin receptor (p75NTR) is a TNF receptor superfamily member that is expressed at high levels during embryogenesis and functions as a pro-apoptotic receptor during the development of neuronal and non-neuronal tissues. $^{1-3}$ The ligand requirements and downstream signaling cascades activated by p75NTR are beginning to emerge and mounting data indicates that p75NTR induces cell death through mechanisms that are distinct from those activated by other pro-apoptotic TNFR superfamily members. p75NTRdependent apoptosis does not involve caspase-8 activation but instead requires activation of Jun kinase (JNK), phos-

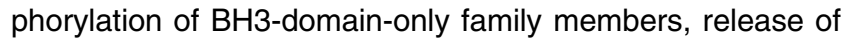
mitochondrial contents, and activation of caspase-9. ${ }^{4-7}$

Independent studies have shown that several members of the MAGE protein family bind to the p75NTR cytosolic region $^{8-10}$ and one of them, termed NRAGE (also known as Maged1 and DIxin) is a potent activator of JNK and inducer of apoptosis. ${ }^{5,11}$ NRAGE is broadly expressed during development and in vitro studies have implicated NRAGE in cellular functions ranging from cell cycle regulation and cell adhesion to transcriptional regulation. ${ }^{12-15}$ We have previously shown that NRAGE is recruited to p75NTR in a neurotrophindependent manner and proposed that NRAGE functions as an adaptor protein that links p75NTR to JNK activation.

In this study, we generated NRAGE knockout mice to assess the physiological role of NRAGE and to specifically test the hypothesis that NRAGE functions as a pro-apoptotic p75NTR adaptor protein in vivo. We demonstrate that NRAGE plays an essential non-redundant role in developmental apoptosis of sympathetic neurons in vivo. In primary sympathetic neurons derived from NRAGE knockout mice, apoptosis induced by brain-derived neurotrophic factor (BDNF), a p75NTR ligand, is sharply attenuated. We confirmed these results in rat sympathetic neurons in which NRAGE levels were reduced by RNA interference. We also report that NRAGE knockout mice exhibit defects in hair follicle catagen that mimic those previously reported for p75NTR knockouts. Together, these findings establish that NRAGE functions as a pro-apoptotic adaptor protein that mediates p75NTR apoptotic-signaling events in vivo. In addition, we show that NRAGE knockout mice, but not p75NTR knockout mice, have a severe defect in motoneuron apoptosis, suggesting that NRAGE may function as an adaptor protein for pro-apoptotic receptors distinct from p75NTR. Taken together, these data demonstrate that NRAGE is a versatile proapoptotic protein required for developmental apoptosis.

\section{Results}

NRAGE knockouts are viable and fertile. To determine if NRAGE is required for p75NTR-induced apoptosis in vivo, we generated mice in which exons 4-12 of the NRAGE gene

\footnotetext{
${ }^{1}$ URPHYM (Unité de Recherches en Physiologie Moléculaire), FUNDP School of Medicine, University of Namur, Namur, Belgium; ${ }^{2}$ Ludwig Institute for Cancer Research, Brussels Branch, Brussels, Belgium; ${ }^{3}$ Montreal Neurological Institute, McGill University, Montreal, Quebec, Canada; ${ }^{4}$ Department of Biochemistry, Vanderbilt University, Nashville, TN, USA and ${ }^{5}$ Institut de Biologie du Développement de Marseille Luminy, Campus de Luminy, Marseille, France

*Corresponding author: PA Barker, Montreal Neurological Institute, McGill University, 3801 University Avenue, Montreal, Quebec, Canada H3A2B4.

Tel: + 514398 3064; Fax: + 514398 5214; E-mail: phil.barker@mcgill.ca

${ }^{6} \mathrm{PAB}$ and ODB are co-senior authors

Keywords: BDNF; motoneuron; sympathetic neuron; Mage; Necdin; apoptosis

Abbreviations: BDNF, brain-derived neurotrophic factor; HF, hair follicle; JNK, jun kinase; MAGE, melanoma antigen-encoding gene; MHD, MAGE homology domain; NRAGE, neurotrophin-interacting MAGE homologue; P75NTR, p75 neurotrophin receptor; SCG, superior cervical ganglion

Received 18.12.07; revised 09.6.08; accepted 30.7.08; Edited by C Duckett; published online 05.9.08
} 
a NRAGE/Maged1 locus



Deleted allele

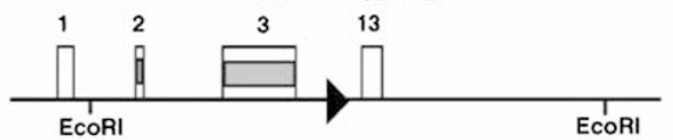

b



C



d

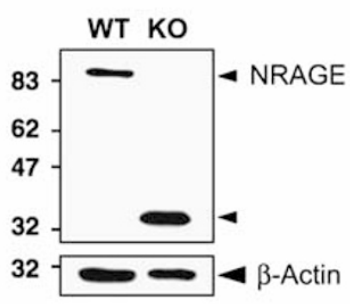

Figure 1 Targeted mutation of NRAGE/Maged1: (a) Genomic organization of the NRAGE/Maged1 locus and schematic representation of the inactivation procedure. The conditional allele (Maged $1^{\text {tm } 1 \text { Urfm })}$ ) and the deleted allele (Maged $\left.1^{\text {tm } 1.1 U r f m}\right)$, generated after Cre-mediated recombination, are shown. Filled boxes represent the coding region of NRAGE, the segment encoding the p75NTR-binding domain is in black. The loxP sites are represented by solid triangles. (b) Southern blot analysis of DNA isolated from wild-type ES cells and from ES cells that have undergone gene replacement (EcoRI/Pagl double digestion). (c) PCR analysis of DNA isolated from tail biopsies of NRAGE knockout, heterozygous and wild-type mice. PCR amplification with primers 93 and 76 gives rise to a 940-bp product specific of the deleted allele (Maged $t^{\text {tm1.1Urim }}$ ) whereas amplification with primers 92 and 172 produces a 270-bp fragment specific of the wild-type allele. (d) Immunoblot analysis of protein lysates from NRAGE knockout and wildtype E13.5 brains

were deleted (Figure 1a). The deleted region contains the conserved MAGE homology domain (MHD) as well as the NRAGE segment that binds p75NTR. Southern blotting and PCR confirmed that the NRAGE gene was successfully targeted (Figure $1 \mathrm{~b}$ and $\mathrm{c}$ ) and immunoblotting of brain lysates demonstrated that full-length NRAGE was absent in the deleted animals. Exons 1-3 of the NRAGE gene remained intact in the deleted animals and we observed the presence of a truncated NRAGE fragment on immunoblots that presumably contained the initiator codon in exon 2 and the subsequent coding sequence in exons 2-3 (Figure 1d). This portion of the NRAGE protein has no obvious protein motifs and is poorly conserved and our analyses assumed that the mice lacking exons 4-12 of the NRAGE gene represent functional knockouts.

NRAGE knockout mice were born with normal Mendelian frequencies when maintained as an inbred line on the C57BI6 strain or when outbred to CD1 animals (Figure 2a). Gross morphological and histopathological features of NRAGE knockout mice were normal (data not shown) and weight gain of NRAGE knockout mice was not different from wild-type littermates (Figure 2b). In vitro studies have raised the possibility that NRAGE may play a role in skeletal development or in cell cycle control yet whole-mount alcian blue/ alizarin red staining did not reveal abnormalities in skeletal development (Figure 2c) and cell cycle control of NRAGE knockout dermal fibroblasts, analyzed by FACS analysis, was indistinguishable from that observed in dermal fibroblasts from wild-type animals (Supplementary Figure S1). We therefore focused our efforts to determine if NRAGE has a function in developmental apoptosis that is regulated by p75NTR.

Hair follicle catagen is defective in NRAGE knockouts. After morphogenesis, hair follicles undergo alternating periods of cell growth (anagen), apoptosis-driven 
a

\begin{tabular}{|c|c|c|c|c|}
\hline & \multicolumn{2}{|c|}{ C57BI/6J } & \multicolumn{2}{|l|}{$\mathrm{N}=258$} \\
\hline & $q_{x x}$ & f $_{x^{*} \times}$ & $\sigma_{X Y}^{x}$ & $\sigma^{x} X^{*} Y$ \\
\hline$q_{X^{*} X \times \sigma^{\top} X Y}$ & $28 \%$ & $25 \%$ & $23 \%$ & $24 \%$ \\
\hline
\end{tabular}

\begin{tabular}{|c|c|c|c|c|}
\hline & \multicolumn{2}{|r|}{ Outbrec } & \multicolumn{2}{|l|}{$\mathrm{N}=272$} \\
\hline & $Q_{x^{*} x}$ & $q^{*} x^{*}$ & $\sigma^{x} X Y$ & $\sigma^{*} x^{*} Y$ \\
\hline$q_{x} \times x \times \sigma^{x} x^{*} y$ & $28 \%$ & $24 \%$ & $24 \%$ & $24 \%$ \\
\hline
\end{tabular}

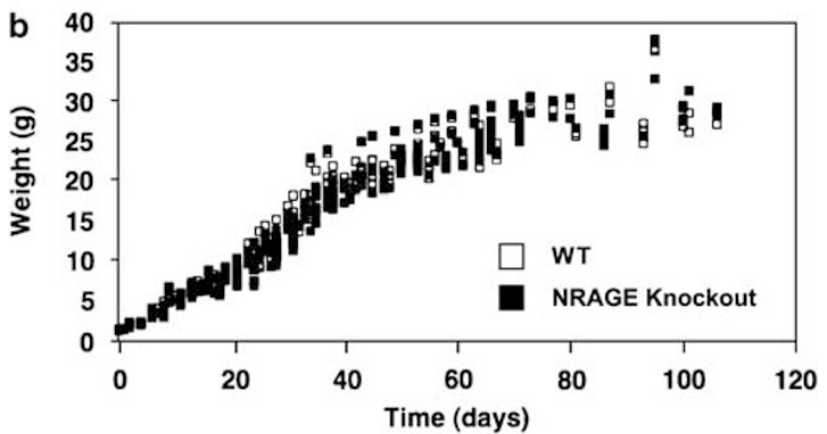

c

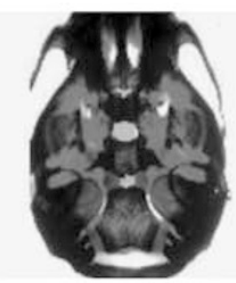

WT

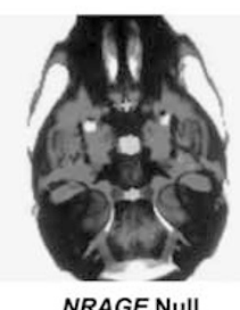

Figure 2 NRAGE deficiency has no impact on viability: (a) The fraction of each genotype generated by crossing heterozygous females $\left(X^{*} X\right)$ with either wild-type $(X Y)$ or hemizygous $\left(X^{\star} Y\right)$ males do not differ from Mendelian predictions on either a pure $\mathrm{C} 57 \mathrm{~B} / 6$ background or in outbred CD1 strains. (b) NRAGE knockout and wildtype littermates display similar weight gain. (c) Alcian blue/alizarin red staining of PO mice did not reveal abnormalities in skeletal development

regression (catagen) and resting (telogen). HF morphogenesis and the first HF catagen and telogen occur with stereotypic developmental timing; in C57BL/6 genetic background, HF morphogenesis is complete by postnatal day 9 (P9), the catagen phase is detected at $\mathrm{P} 17$ and telogen starts 3 days later. ${ }^{16}$ The apoptosis that occurs in the catagen phase relies on neurotrophin-driven activation of p75NTR. ${ }^{17,18}$ During catagen, most cells expressing p75NTR lie in the outer root sheath (ORS) and loss of p75NTR is associated with a decreased apoptosis in those cells. We used immunostaining to analyze the expression of NRAGE and p75NTR proteins in HF undergoing catagen at P18 in C57BL/6 mice. Figure $3 a$ shows that both proteins colocalize in the ORS, the site of p75NTR-dependent apoptosis. To compare NRAGE and p75NTR knockout mice for defects in catagen, we examined the anagen to catagen transition, during the first postnatal HF cycle and after depilation, using the criteria established by Paus and co-workers. ${ }^{16}$ Back skin samples were collected at P17 for the first HF cycle (WT $N=6, \mathrm{KO} N=6$ ) and 19 days post depilation (WT $N=13, \mathrm{KO} N=13$ ). NRAGE-deficient mice showed a profound delay in catagen-dependent dermal thinning, reduction in the hair follicle bulb volume and hair follicle shortening during the first hair follicle cycle (Figure 3b) and after depilation (data not shown). This defect observed in NRAGE nulls did not reflect a delay of HF maturation as the dynamics of HF morphogenesis and growth, analyzed at P1-P2 (WT $N=11$, KO $N=11$ ) and P14 (WT $N=4, \mathrm{KO}$ $N=4$ ), was undistinguishable between $N R A G E$ wild-type and knockout littermates (Supplementary Figure S2 and data not shown). At P21 (WT $N=3, \mathrm{KO} N=3$ ), a light delay in catagen completion was still observed in NRAGE knockout animals when compared with wild-type littermates (Supplementary Figure S2).

p75NTR levels vary during the hair follicle cycle, with highest levels observed in the anagen phase, just before the hair follicle initiates regression. ${ }^{18}$ To establish if NRAGE mRNA and protein levels are regulated in a similar manner, we performed in situ hybridization and immunoblot analyses on dermal sections and lysates. We found abundant NRAGE mRNA expression during all stages of morphogenesis, moderate expression during catagen and no expression during telogen (Figure $3 \mathrm{c}$ and data not shown). Consistent with this, expression levels of NRAGE protein are low in telogen, intermediate in catagen and high during morphogenesis, similar to those previously described for p75NTR (Figure $3 d$ ). The tight co-expression and co-regulation of p75NTR and NRAGE levels, together with the precise phenocopying of catagen defects in p75NTR and NRAGE knockout mice, support the hypothesis that a p75NTRNRAGE signaling pathway regulates hair follicle regression in vivo.

NRAGE is required for p75NTR-dependent apoptosis of sympathetic neurons. NGF and BDNF induce neuronal cell death in vivo by binding p75NTR and activating apoptotic-signaling cascades. This can be replicated in vitro, where BDNF induces p75NTR-dependent apoptosis of sympathetic neurons derived from the superior cervical ganglia (SCG). ${ }^{20,21}$ To determine if NRAGE could have a function in BDNF-induced death of sympathetic neurons, we first examined NRAGE and p75NTR expression levels in SCG during the period of naturally occurring cell death. Figure $4 \mathrm{a}$ shows that NRAGE protein is expressed in postnatal day 4 SCGs and that p75NTR protein expression levels do not change in NRAGE knockout mice. To determine if NRAGE lies on the p75NTR apoptotic cascade, we produced primary cultures of SCG sympathetic neurons from NRAGE knockout mice and wild-type littermates and analyzed the induction of apoptosis in these cells following BDNF exposure. Figure $4 \mathrm{~b}$ shows that in wild-type neurons, BDNF treatment increased sympathetic neuron cell death by approximately fourfold (from 11 to $43 \%$ ). In NRAGE knockout neurons, the effect of BDNF was significantly reduced, with exposure to the ligand causing only a twofold increase in SCG cell death (from 12 to $22 \%$ ). Therefore, we conclude that NRAGE has an important, but not exclusive, function in p75NTR-dependent apoptosis activated by BDNF exposure.

To confirm these results, we produced lentivirus-expressing miRNA that targets NRAGE mRNA for destruction and asked whether depletion of NRAGE reduces BDNF-induced killing in 
a



NRAGE

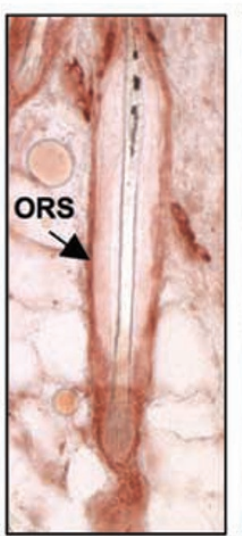

P75NTR

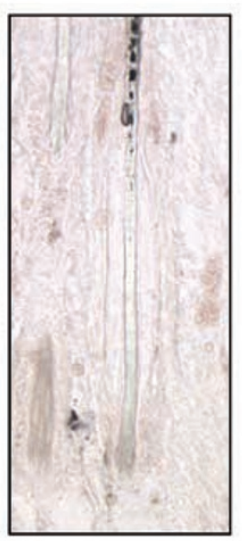

Control b

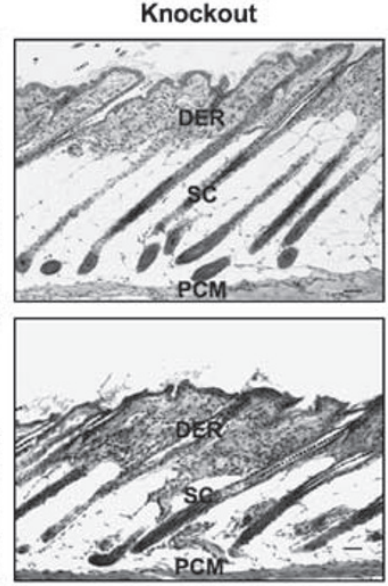

C

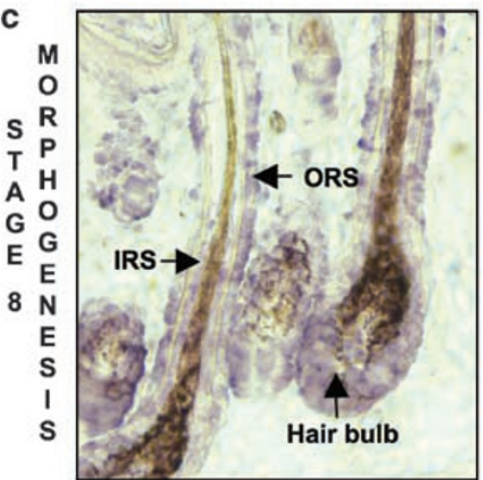

d

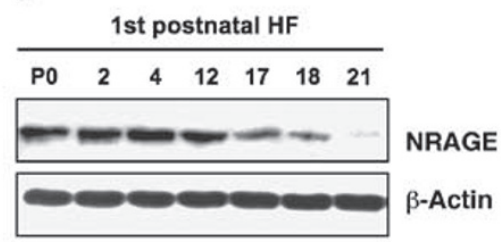

Figure 3 Hair follicle catagen is retarded in NRAGE knockout mice: (a) Immunocytochemistry performed on serial back skin sections of a P18 C57BL/6 mouse showing expression of NRAGE and p75NTR protein in the outer root sheath of a hair follicle on catagen VI. (b) Retardation of hair follicle regression in P17 NRAGE knockout mice resembles that observed in P17 p75NTR knockout mice. Note the thickening of the dermis in each of the null strains. (c) In situ hybridization using an NRAGE probe labeled with digoxygenin ${ }^{19}$ showing NRAGE RNA expression in the inner root sheath, the outer root sheath and hair bulb within a hair follicle of a P4 C57BL/6 mouse. (d) NRAGE protein expression at different stages of the first hair follicle cycle. Scale bar in B is $50 \mu \mathrm{m}$. IRS, inner root sheath; ORS, outer root sheath; DER, dermis; SC, subcutis; PCM, panniculus carnosus muscle

primary rat sympathetic neurons. When sympathetic neurons infected with lentivirus that target NRAGE mRNA were exposed to BDNF, cell death was dramatically reduced, both at 48 and $72 \mathrm{~h}$ after BDNF exposure was initiated (Figure 4c and Supplementary Figure S3). Identical results were obtained using two distinct lentiviruses that target distinct NRAGE regions whereas a lentivirus expressing a control miRNA had no effect on BDNF-induced killing.

Activation of JNK is required for sympathetic neurons apoptosis induced by p75NTR and we therefore assessed if loss of p75NTR or NRAGE caused a reduction in BDNFinduced JNK activation in primary sympathetic neurons. Primary rat sympathetic neurons were infected with lentivirus targeting p75NTR or NRAGE and 6 days later, these cells were examined for BDNF-induced JNK activation. Figure 4d shows that lentivirus targeting p75NTR dramatically reduced levels of the receptor ( $>90 \%$ ) and that, as expected, BDNFinduced activation of JNK was strongly reduced in these cells. Lentivirus targeting NRAGE sharply reduced NRAGE protein levels and, importantly, loss of NRAGE resulted in an almost complete loss of BDNF-induced JNK activation. We conclude that NRAGE is a key element linking p75NTR to JNK activation in sympathetic neurons. As above, identical results were obtained using lentivirus that target different NRAGE regions, and the control miRNA had no effect on BDNFinduced JNK activation.

p75NTR has an important role in the developmental apoptosis of sympathetic neurons within the SCG. In mice, the pool of SCG neurons normally declines by more than $50 \%$ over the first 3 weeks of life and previous studies have shown that this decrease is significantly attenuated in p75NTR knockout animals. ${ }^{20,22}$ To determine if NRAGE has a role in the developmental death of sympathetic neurons in vivo, we performed neuronal counts on SCGs from wild-type and NRAGE knockout mice. At E17, before programmed cell death takes place in these ganglia, the number of sympathetic neurons was essentially identical in wild-type and NRAGE knockout littermates. The peak of cell death in the SCG occurs in early postnatal stages and at P3, we found that the number of surviving sympathetic neurons was much higher in NRAGE knockout animals. Between P3 and P23, when most programmed cell death has normally ended, the number of sympathetic neurons in the NRAGE null animals had dropped 
a

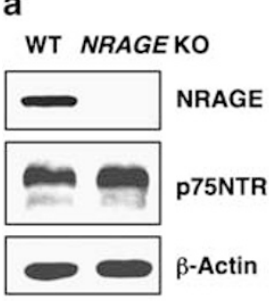

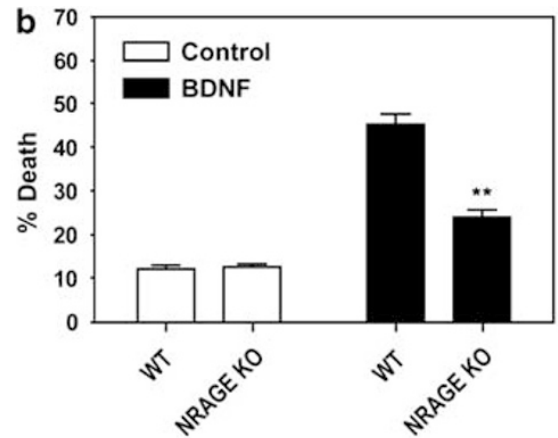

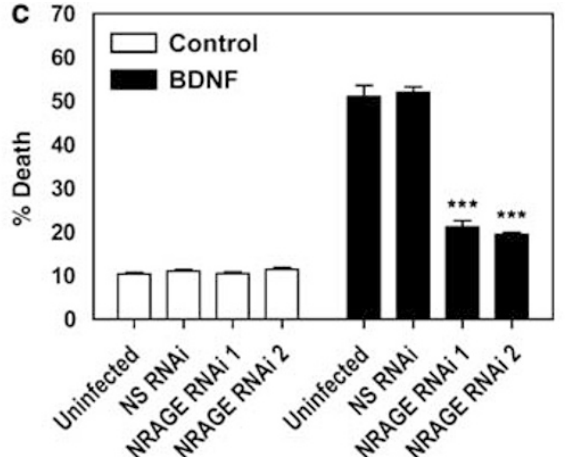

d NS p75 NRAGE1 NRAGE2

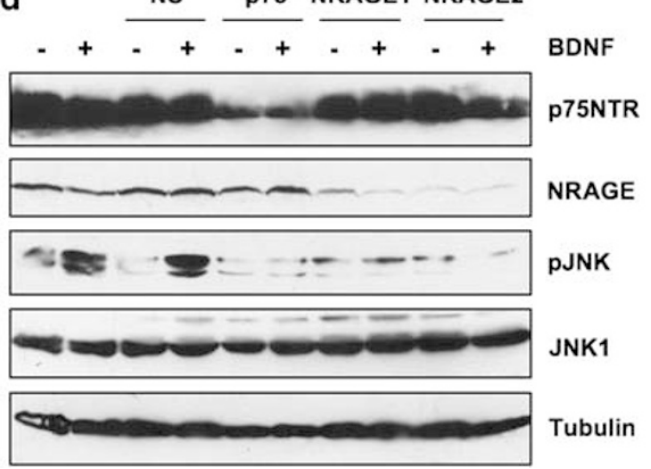

e

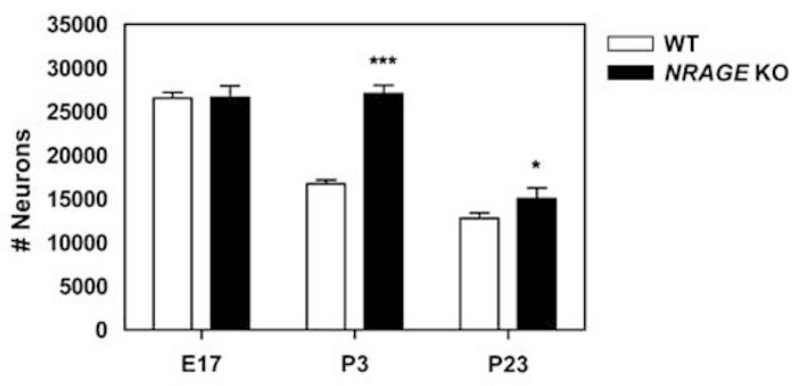

Figure 4 NRAGE knockout SCG sympathetic neurons are deficient in p75NTR-induced apoptosis (a) NRAGE and p75NTR expression in lysates from P4 SCGs of NRAGE knockout and wild-type control pups was determined by immunoblot. (b) Sympathetic neurons derived from NRAGE null animals or from wild-type littermates were exposed to BDNF and apoptotic nuclei were quantified $48 \mathrm{~h}$ later. Approximately 50-70 neurons were counted for each condition. The experiment was performed in triplicate. ${ }^{* *} P=0.0022$. (c) Rat sympathetic neurons were infected with lentivirus-expressing miRNA that targets NRAGE or were infected with a negative control lentivirus-expressing miRNA that does not target a mammalian protein (NS RNAi), then cultured for 5 days. Neurons were then exposed to BDNF for $72 \mathrm{~h}$ and apoptotic nuclei were quantified. ${ }^{\star \star \star} P<0.0001$. (d) Rat sympathetic neurons were infected as in (c) but were also infected with a lentivirus expressing miRNA that targets $p 75 N T R$. Six days later, neurons were exposed to BDNF for $1 \mathrm{~h}$, lysed and analyzed by immunoblot, as indicated. (e) Number of neurons within the superior cervical ganglia were quantified in NRAGE null and wildtype littermates at E17 $(n=3)$, at P3 $(n=5)$ and at P23 (NRAGE knockout: $n=8$, wild-type controls: $n=10) .{ }^{\star \star \star} P<0.0001,{ }^{*} P=0.05$. For $\mathbf{b}, \mathbf{c}$ and $\mathbf{e}$, significance values were calculated using unpaired Student's t-tests. Error bars represent S.E.M.

substantially but they were still significantly higher than in wildtype littermates (Figure 4e). We conclude that NRAGE is required for normal developmental cell death of SCG sympathetic neurons.

Neuronal growth is normal in NRAGE knockout mice. Mice rendered null for p75NTR have defects in neuronal growth that are manifest early in development. To assess whether NRAGE contributes to p75NTR-dependent neuronal growth, we compared wild-type, p75NTR knockout and NRAGE knockout embryos for neuronal growth defects at E11.5, E12.5 and E13.5, using whole-mount immunostaining for $\beta 3$-tubulin to mark neuronal projections. As previously reported, ${ }^{23-25}$ p75NTR knockout embryos displayed profound defects in several neuronal projections at these stages; as an example, Figure 5 shows that the ophthalmic branch of the trigeminal ganglion is severely truncated in p75NTR knockout animals at E12.5. In contrast, neuronal projections in NRAGE knockout embryos were indistinguishable from wild-type littermates at all stages examined (Figure 5 and data not shown). Therefore, although NRAGE is involved in p75NTR-dependent apoptosis, it is dispensable for effects on neuronal growth.
NRAGE knockout mice display defects in developmental apoptosis of motoneurons. p75NTR is expressed at high levels in developing motoneurons ${ }^{26}$ and some studies have suggested that NGF facilitates p75NTR-dependent developmental apoptosis in spinal motoneurons. ${ }^{27,28}$ However, the spinal cord motoneuron pool present in wildtype and $p 75 N T R$ knockouts is identical at P7, suggesting that the receptor is not involved in the programmed cell death of these cells in vivo. ${ }^{29}$ Whether p75NTR has a function in motoneuron apoptosis earlier in development has not been examined and we therefore used the whole-mount spread method of Yamamoto and Henderson ${ }^{30}$ to ask whether p75NTR or NRAGE deletion alters developmental death of motoneurons. We found that mice lacking p75NTR do not have a defect in developmental motoneuron death but surprisingly, motoneuron apoptosis was sharply reduced in NRAGE null embryos (Figure 6a). Quantification of motoneuron apoptosis in the lumbar region of E13.5 embryos revealed that developmental apoptosis of motoneurons was reduced by $42 \%$ in NRAGE knockout mice compared with wild-type littermates, a highly significant effect (Figure 6b; $P=0.008$ ). These data indicate that NRAGE functions in a p75NTR-independent manner to facilitate motoneuron cell death in vivo. 


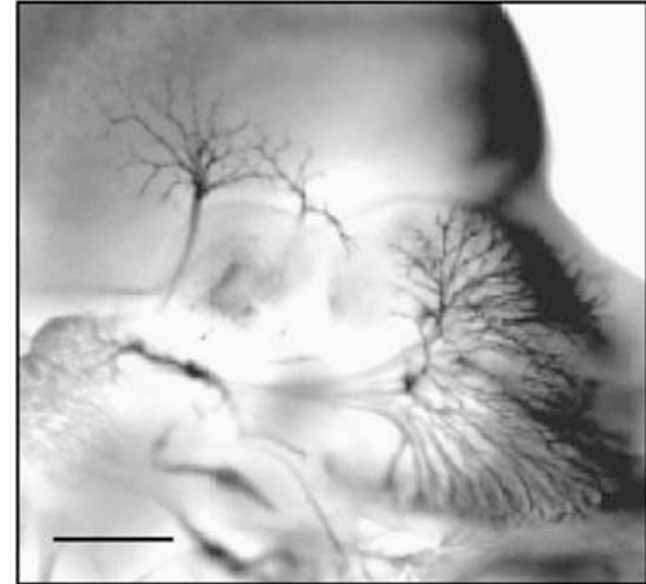

WT

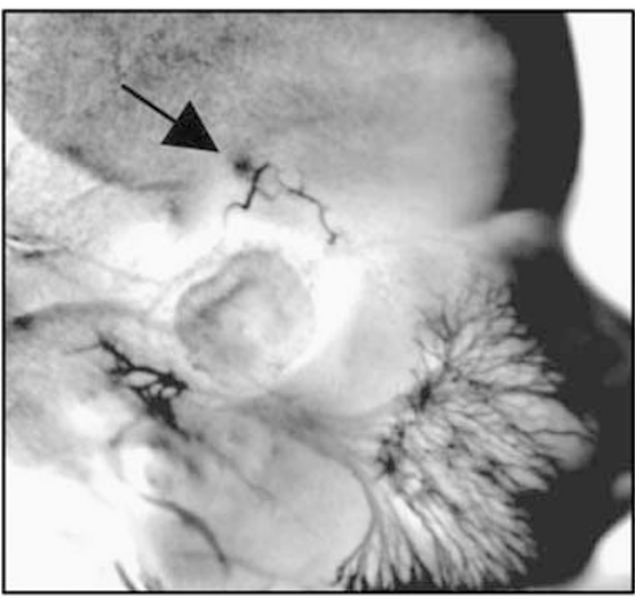

p75NTR KO

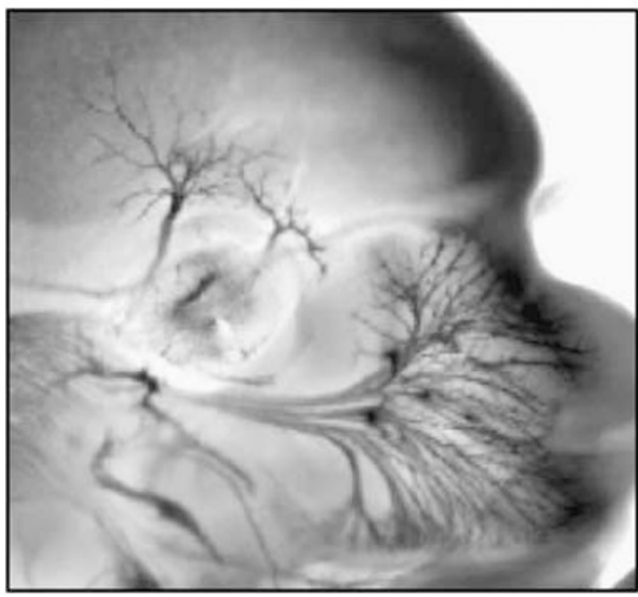

NRAGE KO

Figure 5 Abnormal outgrowth of the ophthalmic branch of the trigeminal ganglion in p75NTR knockout mice but not in NRAGE knockout mice. Whole-mount immunostaining of wild-type, p75NTR knockout and NRAGE knockout embryos with TuJ1, a monoclonal antibody raised against the neuron-specific marker $\beta 3$-tubulin. Arrow indicates growth defect in the ophthalmic branch of the trigeminal ganglion in p75NTR knockout embryos. Scale bar: $0.35 \mathrm{~mm}$

\section{Discussion}

Over 25 MAGE genes are expressed in the human genome but their functions remain poorly understood. ${ }^{15,31}$ MAGE proteins are characterized by a $\sim 200$ amino acid region, of

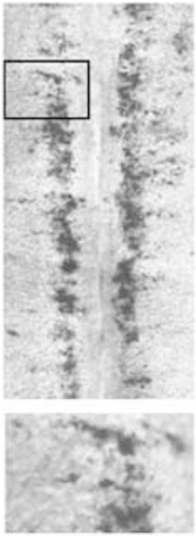

WT

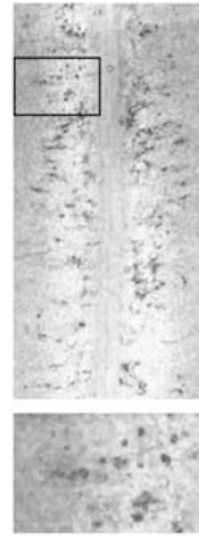

Knockout

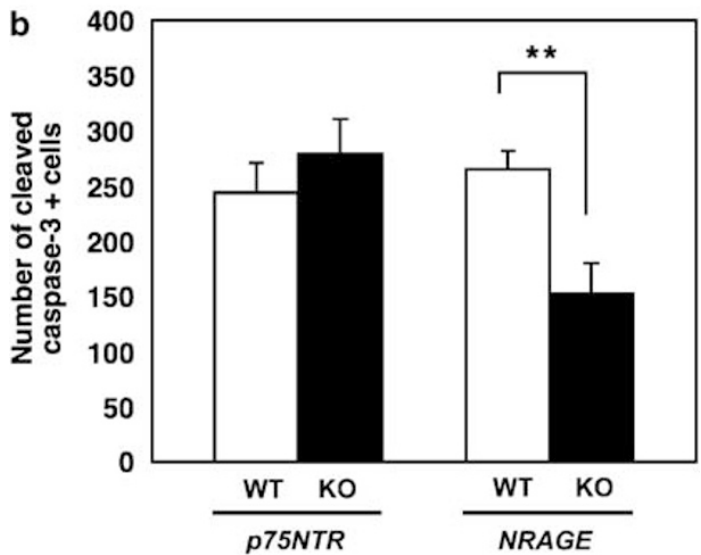

Figure 6 NRAGE, but not p75NTR, is required for motoneuron apoptosis in vivo. (a) Whole-mount cleaved caspase-3 immunostaining of the lumbar region of E13.5 spinal cords from wild-type and NRAGE knockout embryos. Bottom panels represent magnifications of the inset regions. (b) Quantification of lumbar spinal motoneuron apoptosis. NRAGE knockout: $n=4$, wild-type littermates: $n=5$; p75NTR knockout: $n=8$, wild-type littermates: $n=12 .{ }^{* \star} P=0.0081$. $P$-value was calculated using unpaired, two-tailed Student's t-test. Error bars represent S.E.M.

unknown function, termed the MAGE homology domain. ${ }^{15}$ On the basis of their expression pattern, MAGE genes fall into two categories: Type I MAGE genes are activated in a wide variety of tumors but are silent in normal tissues, with the exception of testis and, in some cases, the placenta. Type II MAGE genes are broadly expressed in embryonic and adult somatic tissues. Most MAGE genes are single exon-encoding genes, likely formed by retrotransposition events, and are poorly conserved during mammalian evolution. NRAGE (also known as Maged1 and D/xin) is a Type II MAGE gene that shows strong phylogenetic conservation with the unique MAGE gene found in Drosophila and other lower species, ${ }^{32,33}$ both with regard to its amino acid sequence and its genomic structure. Thus, NRAGE is likely to perform a fundamental cellular function, which has been phylogenetically conserved.

NRAGE has been implicated in a large number of cellular processes. In vitro studies have indicated that NRAGE binds DIx and Msx transcription factors and modulates their transcriptional activity ${ }^{13,14,34}$ and that NRAGE regulates p53-dependent transcription. ${ }^{35}$ NRAGE has also been shown to alter cell cycle progression, ${ }^{36}$ to disrupt E-cadherin- 
dependent adhesion ${ }^{12}$ and to facilitate p38 MAPK activation in response to bone morphogenetic proteins. ${ }^{37}$ Finally, NRAGE binds to the p75NTR intracellular domain and facilitates apoptosis following neurotrophin exposure, suggesting that NRAGE is an adaptor protein that links p75NTR to the apoptotic cascade. ${ }^{5,11,38}$ Our goal in this study was to test the hypothesis that NRAGE functions as an adaptor protein that is required for apoptosis induced by the p75 neurotrophin receptor ( $\mathrm{p} 75 \mathrm{NTR})$ in vivo.

NRAGE knockout mice are fertile, grossly normal and show no obvious defects at birth or in adulthood. Although NRAGE has been implicated in cell proliferation and in transcriptional events that regulate skeletal development, our analyses did not reveal obvious defects in cell cycle control or in the skeletal system. Given the high degree of homology between NRAGE and the other MAGED genes, it is possible that compensatory effects from other alleles dampen an NRAGE loss of function phenotype. The targeted allele produced in this study lacked exons 4-12 (which encodes the MHD as well as the portion of NRAGE that binds p75NTR) but left exons 1-3 intact. The fragment encoded by these exons contain no identifiable protein motifs and does not bind to p75NTR but we cannot rule out the possibility that this fragment has cellular consequences that may ameliorate or exacerbate the consequences of NRAGE gene deletion.

p75NTR has an important function in the developmental cell death of sympathetic neurons. SCGs in mice lacking p75NTR have considerably more sympathetic neurons during and shortly after the period of naturally occurring cell death than their wild-type counterparts. ${ }^{20}$ We performed neuronal counts of the SCG in NRAGE knockouts and in wild-type littermates and found that NRAGE null mice exhibit a defect in sympathetic neuron apoptosis very similar to that observed in the p75NTR mice. For example, recent results from Ginty and co-workers have indicated that at P3, wild-type mice have about 15500 SCG neurons whereas p75NTR nulls have approximately 24000 neurons; we report that P3 wild-type animals have 17000 SCG neurons whereas the NRAGE null mice have approximately 27000 neurons. At P21, Deppmann et al. ${ }^{22}$ report that wild-type SCGs have about 12000 neurons whereas p75NTR nulls have about 15000 neurons and we report that wild-type animals possess 12500 versus 15000 neurons in NRAGE nulls. Therefore, SCG survival defects are precisely phenocopied in p75NTR and NRAGE animals, consistent with the hypothesis that a p75NTR-NRAGEsignaling cascade is required for normal SCG neuronal death in vivo.

To confirm that NRAGE functions in a p75NTR apoptotic cascade that is activated by neurotrophin, we generated primary sympathetic neuron cultures from NRAGE knockout animals and showed that apoptosis induced by the proapoptotic ligand, BDNF, was severely attenuated in these cells. Because NRAGE knockout cells harbor a fragment of the NRAGE gene that may skew these results, we confirmed these findings in primary rat sympathetic neurons, using lentivirus-driving miRNA-targeting NRAGE to knockdown NRAGE gene products. Results obtained using this approach were entirely consistent with data derived from the NRAGE null neurons and we conclude that NRAGE normally has an important function in BDNF-induced apoptosis of sympathetic neurons. It is noteworthy that NRAGE reduces but does not completely block p75NTR-dependent cell death in vitro and therefore independent pro-apoptotic pathways may lie downstream of this receptor. NRIF, has been shown to have an important function in BDNF-induced sympathetic neuronal death in vitro and BDNF may therefore activate p75NTRdependent apoptosis in sympathetic neurons through the actions of at least two adaptor proteins, NRAGE and NRIF. We had previously shown that NRAGE overexpression can activate $\mathrm{JNK}^{11}$ and we therefore tested whether NRAGE knockdown attenuated BDNF-induced JNK activation in sympathetic neurons. We found that knockdown of either NRAGE or p75NTR strongly inhibited JNK activation induced by BDNF, indicating that a p75NTR-NRAGE signaling cascade is required for BDNF-dependent JNK activation in SCG neurons.

We have shown that NRAGE knockout animals show delays in hair follicle catagen that are remarkably similar to those observed in mice lacking p75NTR, ${ }^{18,39}$ suggesting that NRAGE lies on the p75NTR catagen cascade in this organ. Expression analyses showed that NRAGE and p75NTR are co-expressed in the hair follicle compartment where p75NTRdependent apoptosis occurs and that NRAGE and p75NTR are similarly regulated in this tissue, with increased expression just before catagen. Given that a recent study has shown that proNGF expression peaks during catagen, ${ }^{40}$ it seems likely that the defect in catagen observed in NRAGE knockouts reflects loss of a proNGF-p75NTR-NRAGE signaling cascade.

Approximately one-half of spinal motoneurons initially produced are removed by programmed cell death during development. ${ }^{30}$ p75NTR is highly expressed in these cells at this stage and several studies have addressed its ability to induce motoneuron cell death. Sedel et al. ${ }^{28}$ found that motoneurons in dissected neural tubes underwent NGF apoptosis that was blocked by antibodies to p75NTR and Wiese and colleagues (1999) showed that administration of exogenous NGF increases p75NTR-dependent death of motoneurons, particularly in injured facial motoneurons. ${ }^{27,28}$ More recently, Pehar et al. ${ }^{41}$ have shown that NGF can induce apoptosis of normal spinal motoneurons but not those derived from p75NTR knockout mice. Despite these intriguing in vitro findings, Murray et al. ${ }^{29}$ found that the pool of spinal cord motoneurons present at P7 is completely normal in p75NTR knockout animals. We examined motoneuron apoptosis during the period of naturally occurring cell death and similarly, found no evidence for a defect in motoneuron apoptosis in p75NTR nulls.

Surprisingly, we found that NRAGE knockout animals show a major defect in the developmental death of spinal motoneurons. The mechanism by which NRAGE participates in programmed cell death in motoneurons are unknown and, although we cannot rule out the possibility that the NRAGE fragment present in knockout animals results in a toxic gainof-function in motoneurons, a more interesting possibility is that NRAGE functions as an adaptor protein for receptors other than p75NTR to mediate this effect. One interesting candidate for this role is the UNC5A netrin receptor. UNC5A directly binds to NRAGE (through a domain lacking in the NRAGE knockout) and UNC5A induces apoptosis in cultured 
cells through an NRAGE-dependent pathway. ${ }^{42}$ Intriguingly, UNC5A knockout mice have defects in the developmental apoptosis of motoneurons ${ }^{43}$ similar to those reported here for the NRAGE knockout. The defects in motoneuron apoptosis reported in UNC5A knockouts ${ }^{43}$ are not phenocopied in Netrin-1 knockouts and the ligand responsible for this effect has not been identified. Identifying the ligand(s) required Unc5A-induced motoneuron apoptosis and assessing the role of NRAGE in this cascade are important future challenges.

To conclude, we have shown that NRAGE is required for p75NTR-dependent apoptosis in sympathetic neurons and required for normal hair follicle catagen, an apoptotic event that is dependent on p75NTR. In addition, we show that NRAGE induces developmental apoptosis of motoneurons, perhaps through functional interactions with other proapoptotic receptors. Taken together, these studies demonstrate that NRAGE is a p75NTR adaptor protein that facilitates programmed cell death in vivo.

\begin{abstract}
Materials and Methods
Animal strains. NRAGE/Maged 1 knockout mice are described below. p75NTR knockout mice are exon 3-targeted mice originally generated by Dr. Kuo Fee Lee and co-workers. ${ }^{44}$ The morning on which a vaginal plug was observed was considered as E0.5; the day of birth was considered P0. All animal procedures were performed under the guidelines of the Institutional Animal Ethics Committees of the University of Louvain (Belgium), University of Namur (Belgium), IBDM (France) and McGill University (Canada).
\end{abstract}

Generation of NRAGE/Maged1-deficient mice. A conditional allele of NRAGE was generated by homologous recombination in ES cells using standard gene targeting techniques. A 7.5-kb EcoRl genomic fragment served as a template for the targeting vector. A loxP site was introduced into a unique $\mathrm{Ncol}$ site located in intron 12 by inserting a floxed PGK-Neomycin resistance cassette in Cre-expressing E. coli cells. The same PGK-Neo cassette was then inserted into an Avrll site located in intron 3 to generate the targeting construct. The vector was linearized using Notl, electroporated in E14 ES cells and gene replacement was analysed by PCR (primer 168: $5^{\prime}$-GTCCCTTCACCCTGGATTCGG-3' and primer 212: $5^{\prime}$-CGAAGTTATACGCTCTCCTGAG- $3^{\prime}$ for the $5^{\prime}$ side; and primer 90: $5^{\prime}$-TGAC TGGACTGCACAGTT- $3^{\prime}$ and primer 76: $5^{\prime}$-CATGCCACTCTCAGTCAACAGG- $3^{\prime}$ for the $3^{\prime}$ side) and confirmed by Southern blot analysis (EcoRI/Pagl double digestion) using a probe covering nucleotides 70-642 (AF319975). The Neomycin gene was removed by transient expression of Cre after electroporation of a plasmid containing a PGK-Cre gene and a puromycin-resistance cassette. After a short selection $(24 \mathrm{~h})$ with puromycin $(2 \mu \mathrm{g} / \mathrm{ml})$ and cloning by dilution, ES clones containing the conditional allele (Maged $1^{\text {tm } 1 \text { Urfm }}$ ) were identified by PCR (primer 93: $5^{\prime}$-GACCAGCGCAGGTATCTC-3' and primer 172: $5^{\prime}$-TCTCAGATTAGGTGA GGGTCG-3'). Chimeric males were obtained from three independent Maged1 ${ }^{\text {tm1 } 1 \text { rifm }}$ ES clones after injection into CD1 blastocysts (Eurogentec, Belgium). Heterozygous Maged $1^{\text {tm } 1 \text { Urfm }}$ females were bred with Cre deleter males to generate females heterozygous for the knockout mutation. Homozygous males and females were obtained by subsequent crossings. Absence of NRAGE was confirmed by western blot analysis (anti-NRAGE, 1:1000 ${ }^{38}$ ). All phenotypic analysis was performed on mice that had been backcrossed to the C57BL/6 genetic background for a minimum of six generations. Genotyping was performed by PCR using primers 93 and 76 to detect the knockout allele; and primer 92: $5^{\prime}-\mathrm{CA}$ GTGATCTGGCCAAACC- $3^{\prime}$ and primer 172 to detect the wild-type allele.

Histopathology and necropsy. Standard phenotyping was carried out at the 'Institut Clinique de la Souris' (ICS, Strasbourg, France), on adult NRAGE knockout males with age-matched wild-type controls. The phenotyping battery included macroscopic inspection of organs, organ weights (liver, kidney, spleen, heart, visceral fat), total body weight and histopathological evaluation of organs (integument, cardiovascular, respiratory, immune/hematopoietic, digestive tract, digestive organs, urogenital, musculoskeletal, endocrine system, central nervous system, peripheral nervous system and sensory organs). Analysis of skeletons was performed on newborn mice following standard alcian blue and alizarin red staining.
Cell cycle analysis. Mouse embryonic fibroblasts were isolated from E13.5 NRAGE knockout and wild-type littermates. Cells were synchronized to $\mathrm{G}_{0}$ phase by serum starvation for $48 \mathrm{~h}$, then allow to enter the cell cycle by adding serum to the medium. Analysis of relative cellular DNA content was analyzed using propidium iodide and flow cytometry following standard protocols.

Hair follicle analysis. Expression of NRAGE during the first hair follicle cycle was analyzed by in situ hybridization and immunoblot on dermal cryosections and lysates from C57BL/6 mice at P0, P2, P4, P8, P12, P17, P18 and P21 following standard protocols. For the immunostaining analysis, $\mathrm{P} 18$ back skin samples were immersion fixed in $4 \%$ paraformaldehyde (PFA) in phosphate-buffered saline (PBS) for $4 \mathrm{~h}$, left overnight in $30 \%$ PBS-sucrose, and embedded in OCT. Here, $15-\mu \mathrm{m}$ thick sections were serially cut and mounted on superfrost ${ }^{\mathbb{R}} /$ Plus slides (Fisherbrand). Primary antibodies against NRAGE and p75NTR ${ }^{38}$ were used at a dilution of $1 / 100$ following standard protocols. For the analysis of the first hair follicle cycle, back skins from NRAGE knockout and wild-type littermates were harvested at P1-P2, P14, P17 (two p75NTR knockout and two wild-type littermates used as control) and P21. For the anagen-catagen transition after HF cycle induction, 6 - to 8-week-old male mice were depilated with a wax and rosin mixture and skin samples were harvested 19 days post depilation. All skin samples were fixed either in $4 \%$ PFA or Bouin's fixative, embedded in paraffin and sectioned parallel to the vertebral line to obtain longitudinal HF sections.

\section{Sympathetic neurons analysis}

Apoptosis assays. Primary sympathetic neurons were isolated from P6 SCG of NRAGE knockout and wild-type mice and cultured as previously described. ${ }^{45}$ Neurons were maintained in $20 \mathrm{ng} / \mathrm{ml}$ of NGF (Harlan) for 2 days, then NGF was removed, the cells rinsed and switched to media containing anti-NGF $(0.1 \mu \mathrm{g} / \mathrm{ml}$, Chemicon International) together with $12.5 \mathrm{mM} \mathrm{KCl}$, to promote survival, with or without the addition of $200 \mathrm{ng} / \mathrm{ml}$ of BDNF for $48 \mathrm{~h}$. Then cells were fixed in $4 \%$ paraformaldehyde and stained with DAPI (Vector Labs) to score the nuclei as apoptotic or non-apoptotic. Approximately 50-70 neurons were counted for each condition. The experiment was performed in triplicate.

Lentiviral infection. p75NTR and NRAGE knockdowns were achieved by infecting rat sympathetic neurons with lentivirus encoding miRNA with sequences directed against rat $p 75 N T R$ or NRAGE mRNA. The miR targeting sequences were designed using the Invitrogen miR prediction algorithm and cloned following the manufacturer's instructions into a variant of the pcDNA6.2/GW-EmGFP-miR vector (Invitrogen) in which the EmGFP has been replaced with mRFP. VSV-G pseudotyped viral particles were produced in 293-T cells and particles were purified by ultracentrifugation, resuspended in DMEM, and the amount of active viral particles was determined by titration on HEK293 T cells. For miR-mediated knockdown, sympathetic neurons were transduced at an $\mathrm{MOI}$ of 10 and analyzed 5-6 days post-transduction. Apoptosis assays were realized by treating the rat cells with BDNF for 48 and $72 \mathrm{~h}$ following the protocol described above. JNK activation was analyzed by stimulating the cell with BDNF $(200 \mathrm{ng} / \mathrm{ml})$ for one hour. The cells were then lysed in NP-40 buffer and immunoblot for $p$-JNK, JNK1, NRAGE, p75NTR and tubulin.

Neuronal counts of SCGs. SCGs from E17, P3 and P23 NRAGE knockout and wild-type littermates were immersion fixed overnight in formalin and embedded in paraffin. Here, 6- $\mu$ m thick sections were serially cut and mounted on superfrost ${ }^{\mathbb{R}} /$ Plus slides (Fisherbrand). Slides were stained with cresyl violet, scanned with Miraxscan scanner (Zeiss) and neuronal numbers were determined by counting nucleoli present in neuronal profiles on every third section for P3 and P23 and every sixth for E17.

Developmental motoneuron death. Apoptosis in spinal cords was analyzed by whole-mount immunohistochemistry using an antibody directed against cleaved caspase-3 (Asp175, Cell Signaling Technologies; 1/200) following a previously described protocol. ${ }^{30}$ For quantification, spinal cords were divided into five regions (cervical, brachial, thoracic, lumbar and sacral) and the number of apoptotic cells was determined in each region. Four NRAGE knockout, five wild-type littermates, eight $p 75 N T R$ knockout and 12 wild-type littermates were analyzed.

Nerve outgrowth. Peripheral innervation was visualized at E11.5, E12.5 and E13.5 by whole-mount immunohistochemistry using a monoclonal antibody (TuJ1) recognizing the neuron-specific $\beta$-tubulin III protein. A minimum of three knockout and three wild-type littermates were observed for each genotype at all stages of development. 
Acknowledgements. We are grateful to Prof. M Herin for help with hair follicle analysis and to $\mathrm{P}$ Auquier, $\mathrm{D}$ Desnoeck, $\mathrm{C}$ Dernoncourt and $\mathrm{R}$ Déom for technical assistance. Dr. Barry Bedell provided valuable help with sympathetic neuron counts and Drs. Peter McPherson and Brigitte Ritter provided the protocol for producing miRNA-expressing lentivirus. This work was supported by Action de Recherche Concertée no 99/03-248 of the Communauté Française de Belgique (to ODB), by the Fonds de la Recherche Scientifique et Médicale (Grant no 3.4527.04) of the Fonds National de la Recherche Scientifique of Belgium (to ODB), and by operating funds from the Canadian Institutes of Health Research (to PAB). MB was supported by the Fonds pour la formation à la Recherche dans I'Industrie et l'Agriculture (FNRS of Belgium), DA was supported by the French Association pour la Recherche sur le Cancer (ARC) and PAB is an Investigator of the Canadian Institutes of Health Research.

1. Roux PP, Barker PA. Neurotrophin signaling through the $p 75$ neurotrophin receptor. Prog Neurobiol 2002; 67: 203-233.

2. Nykjaer A, Willnow TE, Petersen CM. p75NTR-live or let die. Curr Opin Neurobiol 2005; 15: 49-57.

3. Teng KK, Hempstead BL. Neurotrophins and their receptors: signaling trios in complex biological systems. Cell Mol Life Sci 2004; 61: 35-48.

4. Beattie MS, Harrington AW, Lee R, Kim JY, Boyce SL, Longo FM et al. ProNGF induces p75-mediated death of oligodendrocytes following spinal cord injury. Neuron 2002; 36 375-386.

5. Bhakar AL, Howell JL, Paul CE, Salehi AH, Becker EB, Said F et al. Apoptosis induced by p75NTR overexpression requires Jun kinase-dependent phosphorylation of Bad. J Neurosci 2003; 23: 11373-11381.

6. Harrington AW, Leiner B, Blechschmitt C, Arevalo JC, Lee R, Morl K et al. Secreted proNGF is a pathophysiological death-inducing ligand after adult CNS injury. Proc Natl Acad Sci USA 2004; 101: 6226-6230.

7. Wang X, Bauer JH, Li Y, Shao Z, Zetoune FS, Cattaneo E et al. Characterization of a p75(NTR) apoptotic signaling pathway using a novel cellular model. J Biol Chem 2001; 276: 33812-33820.

8. Kuwako K, Taniura H, Yoshikawa K. Necdin-related MAGE proteins differentially interac with the E2F1 transcription factor and the p75 neurotrophin receptor. J Biol Chem 2004; 279: 1703-1712.

9. Salehi AH, Roux PP, Kubu CJ, Zeindler C, Bhakar A, Tannis LL et al. NRAGE, a novel MAGE protein, interacts with the $p 75$ neurotrophin receptor and facilitates nerve growth factor-dependent apoptosis. Neuron 2000; 27: 279-288.

10. Tcherpakov M, Bronfman FC, Conticello SG, Vaskovsky A, Levy Z, Niinobe M et al. The p75 neurotrophin receptor interacts with multiple MAGE proteins. J Biol Chem 2002; 277 49101-49104.

11. Salehi AH, Xanthoudakis S, Barker PA. NRAGE, a p75 neurotrophin receptor-interacting protein, induces caspase activation and cell death through a JNK-dependent mitochondrial pathway. J Biol Chem 2002; 277: 48043-48050.

12. Xue B, Wen C, Shi Y, Zhao D, Li C. Human NRAGE disrupts E-cadherin/beta-catenin regulated homotypic cell-cell adhesion. Biochem Biophys Res Commun 2005; 336: 247-251.

13. Masuda $Y$, Sasaki A, Shibuya H, Ueno N, Ikeda K, Watanabe K. Dlxin-1, a novel protein that binds DIx5 and regulates its transcriptional function. J Biol Chem 2001; 276 5331-5338.

14. Kuwajima T, Taniura H, Nishimura I, Yoshikawa K. Necdin interacts with the Msx2 homeodomain protein via MAGE-D1 to promote myogenic differentiation of $\mathrm{C} 2 \mathrm{C} 12$ cells. J Biol Chem 2004; 279: 40484-40493.

15. Chomez P, De Backer O, Bertrand M, De Plaen E, Boon T, Lucas S. An overview of the MAGE gene family with the identification of all human members of the family. Cancer Res 2001; 61: 5544-5551.

16. Muller-Rover S, Handjiski B, van der Veen C, Eichmuller S, Foitzik K, McKay IA et al. A comprehensive guide for the accurate classification of murine hair follicles in distinct hair cycle stages. J Invest Dermatol 2001; 117: 3-15.

17. Peters EM, Stieglitz MG, Liezman C, Overall RW, Nakamura M, Hagen E et al. p75 Neurotrophin receptor-mediated signaling promotes human hair follicle regression (Catagen). Am J Pathol 2006; 168: 221-234.

18. Botchkarev VA, Botchkareva NV, Albers KM, Chen LH, Welker P, Paus R. A role for p75 neurotrophin receptor in the control of apoptosis-driven hair follicle regression. FASEB $J$ 2000; 14: 1931-1942.

19. Bertrand M, Huijbers I, Chomez P, De Backer O. Comparative expression analysis of the MAGED genes during embryogenesis and brain development. Dev Dyn 2004; 230 $325-334$
20. Bamii SX, Maidan M, Pozniak CD, Belliveau DJ, Aloyz R, Kohn J et al. The p75 neurotrophin receptor mediates neuronal apoptosis and is essential for naturally occurring sympathetic neuron death. J Cell Biol 1998; 140: 911-923.

21. Kenchappa RS, Zampieri N, Chao MV, Barker PA, Teng HK, Hempstead BL et al. Liganddependent cleavage of the P75 neurotrophin receptor is necessary for NRIF nuclear translocation and apoptosis in sympathetic neurons. Neuron 2006; 50: 219-232.

22. Deppmann CD, Mihalas S, Sharma N, Lonze BE, Niebur E, Ginty DD. A model for neuronal competition during development. Science 2008; 320: 369-373.

23. Bergmann I, Priestley JV, McMahon SB, Brocker EB, Toyka KV, Koltzenburg M. Analysis of cutaneous sensory neurons in transgenic mice lacking the low affinity neurotrophin receptor p75. Eur J Neurosci 1997; 9: 18-28.

24. Yamashita T, Tucker KL, Barde YA. Neurotrophin binding to the p75 receptor modulates Rho activity and axonal outgrowth. Neuron 1999; 24: 585-593.

25. Lee K-L, Bachman K, Landis S, Jaenisch R. Dependence on p75 for innervation of some sympathetic targets. Science 1994; 263: 1447-1449.

26. Ernfors $\mathrm{P}$, Henschen $\mathrm{A}$, Olson $\mathrm{L}$, Persson $\mathrm{H}$. Expression of nerve growth factor receptor mRNA is developmentally regulated and increased after axotomy in rat spinal cord motoneurons. Neuron 1989; 2: 1605-1613.

27. Wiese S, Metzger F, Holtmann B, Sendtner M. The role of p75NTR in modulating neurotrophin survival effects in developing motoneurons. Eur J Neurosci 1999; 11: 1668-1676.

28. Sedel F, Bechade C, Triller A. Nerve growth factor (NGF) induces motoneuron apoptosis in rat embryonic spinal cord in vitro. Eur J Neurosci 1999; 11: 3904-3912.

29. Murray SS, Bartlett PF, Cheema SS. Differential loss of spinal sensory but not motor neurons in the p75NTR knockout mouse. Neurosci Lett 1999; 267: 45-48.

30. Yamamoto W, Henderson CE. Patterns of programmed cell death in populations of developing spinal motoneurons in chicken, mouse, and rat. Dev Biol 1999; 1999: 60-71.

31. Barker PA, Salehi A. The MAGE proteins: emerging roles in cell cycle progression, apoptosis, and neurogenetic disease. J Neurosci Res 2002; 67: 705-712.

32. Bischof JM, Ekker M, Wevrick R. A MAGE/NDN-like gene in zebrafish. Dev Dyn 2003; 228: 475-479.

33. Lopez-Sanchez N, Gonzalez-Fernandez Z, Ninobe M, Yoshikawa K, Frade JM. Single mage gene in the chicken genome encodes $\mathrm{CMage}$, a protein with functional similarities to mammalian type II Mage proteins. Physiol Genomics 2007; 30: 156-171.

34. Sasaki A, Masuda Y, Iwai K, Ikeda K, Watanabe K. A RING finger protein Praja1 regulates Dlx5-dependent transcription through its ubiquitin ligase activity for the Dlx/Msx-interacting MAGE/Necdin family protein, Dlxin-1. J Biol Chem 2002; 277: 22541-22546.

35. Wen CJ, Xue B, Qin WX, Yu M, Zhang MY, Zhao DH et al. hNRAGE, a human neurotrophin receptor interacting MAGE homologue, regulates p53 transcriptional activity and inhibits cell proliferation. FEBS Lett 2004; 564: 171-176.

36. Kendall SE, Goldhawk DE, Kubu C, Barker PA, Verdi JM. Expression analysis of a novel p75(NTR) signaling protein, which regulates cell cycle progression and apoptosis. Mech Dev 2002; 117: 187-200.

37. Kendall SE, Battelli C, Irwin S, Mitchell JG, Glackin CA, Verdi JM. NRAGE mediates p38 activation and neural progenitor apoptosis via the bone morphogenetic protein signaling cascade. Mol Cell Biol 2005; 25: 7711-7724.

38. Salehi AH, Roux PP, Kubu CJ, Zeindler C, Bhakar A, Tannis LL et al. NRAGE, a novel MAGE protein, interacts with the p75 neurotrophin receptor and facilitates nerve growth factor-dependent apoptosis. Neuron 2000; 27: 279-288.

39. Botchkareva NV, Botchkarev VA, Chen LH, Lindner G, Paus R A role for 755 neurotrophin receptor in the control of hair follicle morphogenesis. Dev Biol 1999; 216: 135-153.

40. Peters EM, Hendrix S, Golz G, Klapp BF, Arck PC, Paus R. Nerve growth factor and its precursor differentially regulate hair cycle progression in mice. J Histochem Cytochem 2006; 54: 275-288.

41. Pehar M, Cassina P, Vargas MR, Castellanos R, Viera L, Beckman JS et al. Astrocytic production of nerve growth factor in motor neuron apoptosis: implications for amyotrophic lateral sclerosis. J Neurochem 2004; 89: 464-473.

42. Williams ME, Strickland $\mathrm{P}$, Watanabe K, Hinck L. UNC5H1 induces apoptosis via its juxtamembrane region through an interaction with NRAGE. J Biol Chem 2003; 278: 17483-17490

43. Williams ME, Lu X, McKenna WL, Washington R, Boyette A, Strickland P et al. UNC5A promotes neuronal apoptosis during spinal cord development independent of netrin-1. Nat Neurosci 2006; 9: 996-998

44. Lee K-F, Li E, Huber J, Landis SC, Sharpe AH, Chao MV et al. Targeted mutation of the gene encoding the low affinity NGF receptor leads to deficits in the peripheral sensory nervous system. Cell 1992; 69: 737-749.

45. Palmada M, Kanwal S, Rutkoski NJ, Gustafson-Brown C, Johnson RS, Wisdom R et al. cjun is essential for sympathetic neuronal death induced by NGF withdrawal but not by $p 75$ activation. J Cell Biol 2002; 158: 453-461. 